\title{
Growth and Yield of Taro (Colocasia esculenta (L) Schott.) as Affected by Planting Distance
}

\author{
Richard Boampong (D), Samuel Kwasi Boateng, Richard Adu Amoah, Bright Adu Gyamfi (D), \\ Lawrence Misa Aboagye, and Eric Osafo Ansah
}

CSIR-Plant Genetic Resources Research Institute, P.O. Box 7, Bunso, Eastern Region, Ghana

Correspondence should be addressed to Richard Boampong; richardboampong5@gmail.com

Received 4 May 2020; Revised 10 December 2020; Accepted 16 December 2020; Published 29 December 2020

Academic Editor: Mehdi Rahimi

Copyright (C) 2020 Richard Boampong et al. This is an open access article distributed under the Creative Commons Attribution License, which permits unrestricted use, distribution, and reproduction in any medium, provided the original work is properly cited.

\begin{abstract}
Taro, Colocasia esculenta (L) Schott., is a staple food for many people in Africa. Despite the numerous importance of the crop, it still remains an underutilized crop in Ghana with little information on many aspects of the crop, especially agronomic practices. This experiment was conducted to identify the effect of planting distance on growth and yield of two promising taro accessions. The experiment was laid out using the split-plot design arranged in Randomized Complete Block Design (RCBD) with three replications in which accessions (BL/SM/80 and BL/SM/16) were used as main plots and three planting distances $(1 \mathrm{~m} \times 1 \mathrm{~m}$, $1 \mathrm{~m} \times 0.75 \mathrm{~m}$, and $1 \mathrm{~m} \times 0.5 \mathrm{~m}$ ) as subplots. Data were recorded on the $4^{\text {th }}, 8^{\text {th }}, 12^{\text {th }}, 16^{\text {th }}, 20^{\text {th }}, 24^{\text {th }}$, and $28^{\text {th }}$ week after planting (WAP) for growth parameters while yield data were taken at harvest. The results indicated that plant height $(63.4 \mathrm{~cm})$, petiole length $(44.0 \mathrm{~cm})$, number of leaves $(4.7)$, leaf length $(31.7 \mathrm{~cm})$, and width $(20.5 \mathrm{~cm})$ were significantly $(P \leq 0.05)$ higher in closely spaced plants than widely spaced plants at 4 WAP and 8 WAP for petiole length $(70.3 \mathrm{~cm})$ and in the $28^{\text {th }}$ WAP where there was an accession effect on leaf length and number of suckers/plant. The highest corm yield/plant $(0.63 \mathrm{~kg})$ and total corm yield/ha (11.7 t/ ha) in both accessions were achieved by the medium plant spacing $(1 \mathrm{~m} \times 0.75 \mathrm{~m})$ and lower plant spacing $(1 \mathrm{~m} \times 0.5 \mathrm{~m})$, respectively. Accession BS/SM/80 recorded the highest total yield/ha of $13.0 \mathrm{t} / \mathrm{ha}$ for $1 \mathrm{~m} \times 0.5 \mathrm{~m}$ plant spacing. The higher number of suckers (8.1) was recorded by higher spaced plants. From the study, it was seen that growth parameters correlated significantly and positively with yield. It is therefore recommended that farmers in the area and those in similar production areas use a spacing of $1 \mathrm{~m} \times 0.5 \mathrm{~m}$ for optimum growth and yield.
\end{abstract}

\section{Introduction}

Taro, Colocasia esculenta (L) Schott., is a member of the monocotyledonous family Araceae and subfamily Aroideae $[1-4]$. Taro is the most widely cultivated species in the genus Colocasia [5] and it is the fourth most consumed tuber crop in the world [6]. Taro has enormous health benefits which include building a strong immune system, lowering blood pressure, reducing weight gain and fatigue, preventing cell damage, building strong bone, and also supporting thyroid function $[7,8]$. The importance of taro is increasing as a subsistence crop in the rural areas of Ghana and the rise in importance of the crop can be attributed to the price increase in cereals and other root crops such as yam [9]. Most of the taros produced in the rural areas of Ghana are consumed and as such very few find their way into the markets. Despite taro's contribution to food security, especially in Ghana, agronomic research into taro is very recent [9]. The aspects that have not been worked on in detail with regard to Ghanaian taro include plant population, plant spacing, and planting materials. The constraints mentioned above in the taro industry of Ghana can be tackled and if possible solved through research. Ghana is presently the world's third highest producer of taro producing about 1,460,938 tons per annum which accounts for about $14 \%$ of the world's total and 19\% of Africa's total production [10]. The yield of taro is still low in Ghana as a result of poor production practices. There is therefore the need to enhance taro production to 
help alleviate food insecurity and poverty among rural farmers in Ghana. Plant response to spacing varies from species to species and is highly dependent on such environmental conditions as soil characteristics, biotic elements, and climatic conditions of the site [11]. A report according to [12] indicates that production increases as plant spacing decreases until a point is reached when further decrease only leads to a slight increase in production. To maximize production, interception of light by chloroplast must be maximized to enhance photosynthesis upon which yield of crops is totally and directly dependent [13]. Such increase in yield with decrease in plant spacing has been reported in other crops [14]. However, Osundare [15] noted that average corm weight decreases with a decrease in plant spacing. Plant spacing used in taro affects taro growth, corm shape, and taro yield due to competition for soil moisture, nutrients, and light. A lot of taro production in Ghana still relies on old production methods and therefore research into various agronomic practices is needed to improve the production of taro in Ghana. The objective of this study was therefore to determine the effect of plant spacing on the growth and yield of taro which was aimed at identifying high-yielding accessions and the optimum planting space for maximum production of taro.

\section{Materials and Methods}

2.1. Experimental Site. The experiment was carried out at the Council for Scientific and Industrial Research (CSIR), Plant Genetic Resources Research Institute (PGRRI), at Bunso (longitude $6^{\circ} 17^{\prime} \mathrm{N}$, latitude $0^{\circ} 27^{\prime} \mathrm{W}$, and altitude $214 \mathrm{~m}$ above sea level) in the eastern region of Ghana, during the major rainy season in 2018, on taro (Colocasia esculenta (L) Schott.). The major soil type in the study area is mainly red, well-drained, and silty loam. The soils are suitable for the cultivation of both food crops, such as taro, cassava, plantain, yam, cocoyam, and maize, and cash crops like cocoa, coffee, oil palm, citrus, and cola, which are grown in the area. The area has an annual temperature of about $30^{\circ} \mathrm{C}$ and $600-1200 \mathrm{~mm}$ of rain in a year with an average humidity of $80 \%[16]$.

2.2. Planting Materials. Two planting materials (BL/SM/80 and BL/SM/16) at CSIR-Plant Genetic Resources Research Institute which was introduced from Samoa were used for the study. Both accessions are tolerant of the taro leaf blight (TLB) disease and have very good taste.

2.3. Experimental Design. The experimental field was laid out in a split-plot design arranged in Randomized Complete Block Design replicated three times. Two accessions of taro $(\mathrm{BL} / \mathrm{SM} / 80$ and $\mathrm{BL} / \mathrm{SM} / 16)$ were used as main plots and three different planting distances $(1 \times 1 \mathrm{~m}, 1 \times 0.75 \mathrm{~m}$, and $1 \times 0.5 \mathrm{~m})$ as subplots. The land was cleared and stumped before marking out into blocks and plots according to the experimental design. Each plot measured $4 \times 4 \mathrm{~m}^{2}$ with $2 \mathrm{~m}$ between blocks and $1.5 \mathrm{~m}$ between plots in a block. Planting was done just after land preparation using the various planting distances. Weed control was done once a month throughout the study period and no additional inputs (water and fertilizer) were added.

2.4. Data Collection and Analysis. Observations on six plant characters, namely, plant height, petiole length, leaf length, leaf width, number of leaves/plant, and number of suckers/ plant, as well as yield and yield attributes, namely, corm length, corm diameter harvest index, yield/plant, and total yield/ha were recorded. Data was recorded on five randomly selected plants in each plot. Data was subjected to Analysis of Variance (ANOVA) using Statistix version 9 to examine the presence of statistically significant difference among the plant spacing and accessions and means were separated at 5\% probability level $(P \leq 0.05)$ [17].

\section{Results}

3.1. Growth Parameters. Tables 1 and 2 show the growth of taro plants at three different planting distances on two taro accessions throughout the 7-month growing period (28 weeks after planting, WAP). The first 12 WAP (90 days after planting) were characterized by an increase in plant height, petiole length, leaf length, and leaf width (Tables 1 and 2). The number of leaves per plant increased between 4 and 8 WAP with a drop at $12 \mathrm{WAP}$ and again increased from 12 to 20 WAP. The number of suckers per plant increased with WAP, characterized by a sharp increase between 4 and 24 WAP, after which it nearly leveled at $28 \mathrm{WAP}$ (Tables 1 and 2). Plant height, petiole length, number of leaves, leaf length, and width were significantly $(P \leq 0.05)$ higher in closely spaced plants than widely spaced plants at 4 WAP and 8 WAP for petiole length. On the contrary, the number of leaves for widely spaced plants $(1 \mathrm{~m} \times 1 \mathrm{~m})$ was significantly $(P \leq 0.05)$ different from closely spaced $(1 \mathrm{~m} \times 0.5 \mathrm{~m})$ plants at 20 WAP (Table 1).

Among the accessions, significant $(P \leq 0.05)$ higher plant height, petiole length, and number of suckers were seen in accession $\mathrm{BL} / \mathrm{SM} / 80$ at $4 \mathrm{WAP}$. The number of leaves was significant in accession BL/SM/16 at 12 WAP. At 16 WAP, significantly $(P \leq 0.05)$ higher number of leaves was observed in accession BL/SM/16 (Table 2). At 20 WAP, where vegetative growth of taro is said to be at the peak, plants spaced at $1 \mathrm{~m} \times 0.75 \mathrm{~m}$ recorded higher growth values, although these were not significantly different from each other. The number of suckers was significant $(P \leq 0.05)$ in accession $\mathrm{BL} / \mathrm{SM} / 16$ at $24 \mathrm{WAP}$ and at harvest (28 WAP).

The accession by planting distance interactions effect was not significant on plant growth parameters of taro. The result shown in Tables 3 and 4 indicated a trend of increase in plant growth parameters in all the accessions as planting distance was decreased. Plant growth was the highest in BL/MS/80 for plants spaced at $1 \mathrm{~m} \times 0.5 \mathrm{~m}$ at 16 WAP with the exception of the number of leaves which recorded the maximum at $20 \mathrm{WAP}$ with plants spaced at $1 \mathrm{~m} \times 1 \mathrm{~m}$ recording the higher number of leaves. Accession $\mathrm{BL} / \mathrm{MS} / 16$, on the contrary, showed maximum growth at 24 WAP with plants spaced at $1 \mathrm{~m} \times 0.75 \mathrm{~m}$ recording higher plant height. Similar to accession BL/MS/80, 
TABLE 1: Effect of different planting distances on the growth of taro.

\begin{tabular}{|c|c|c|c|c|c|c|c|}
\hline Week & Plant spacing & $\mathrm{PH}(\mathrm{cm})$ & $\mathrm{NL} / \mathrm{P}$ & $\mathrm{LL}(\mathrm{cm})$ & $\mathrm{LW}(\mathrm{cm})$ & NS/P & $\mathrm{PL}(\mathrm{cm})$ \\
\hline \multirow{6}{*}{4} & $1 \mathrm{~m} \times 1 \mathrm{~m}$ & 54.2 & 4.4 & 27.6 & 17.5 & 0.2 & 37.7 \\
\hline & $1 \mathrm{~m} \times 0.75 \mathrm{~m}$ & 59.5 & 4.2 & 30.4 & 19.6 & 0.7 & 41.3 \\
\hline & $1 \mathrm{~m} \times 0.5 \mathrm{~m}$ & 63.4 & 4.7 & 31.7 & 20.5 & 0.8 & 44.0 \\
\hline & CV (\%) & 13.1 & 9.6 & 10.6 & 12.8 & 28.9 & 15.5 \\
\hline & $P$ value & 0.04 & 0.05 & 0.05 & 0.05 & 0.37 & 0.04 \\
\hline & LSD (0.05) & 6.9 & 0.4 & 3.6 & 2.4 & 0.8 & 4.6 \\
\hline \multirow{6}{*}{8} & $1 \mathrm{~m} \times 1 \mathrm{~m}$ & 85.5 & 5.3 & 39.9 & 26.4 & 2.4 & 61.2 \\
\hline & $1 \mathrm{~m} \times 0.75 \mathrm{~m}$ & 91.3 & 5.6 & 41.1 & 27.9 & 3.1 & 64.7 \\
\hline & $1 \mathrm{~m} \times 0.5 \mathrm{~m}$ & 95.8 & 5.7 & 43.2 & 29.4 & 2.9 & 70.3 \\
\hline & CV (\%) & 13.6 & 11.2 & 10.4 & 11.8 & 27.2 & 17.2 \\
\hline & $P$ value & 0.20 & 0.46 & 0.27 & 0.31 & 0.51 & 0.05 \\
\hline & LSD (0.05) & 11.9 & 0.7 & 5.3 & 4.0 & 1.5 & 8.2 \\
\hline \multirow{6}{*}{12} & $1 \mathrm{~m} \times 1 \mathrm{~m}$ & 100.0 & 5.2 & 42.9 & 29.6 & 3.3 & 74.5 \\
\hline & $1 \mathrm{~m} \times 0.75 \mathrm{~m}$ & 101.1 & 5.3 & 44.4 & 30.3 & 4.2 & 78.6 \\
\hline & $1 \mathrm{~m} \times 0.5 \mathrm{~m}$ & 106.2 & 4.9 & 46.3 & 31.1 & 4.1 & 83.1 \\
\hline & CV (\%) & 15.3 & 7.2 & 9.9 & 12.1 & 26.1 & 17.8 \\
\hline & $P$ value & 0.67 & 0.42 & 0.39 & 0.69 & 0.39 & 0.24 \\
\hline & LSD (0.05) & 15.9 & 0.5 & 5.3 & 4.0 & 1.5 & 10.8 \\
\hline \multirow{6}{*}{16} & $1 \mathrm{~m} \times 1 \mathrm{~m}$ & 101.8 & 5.9 & 42.5 & 28.2 & 5.1 & 76.2 \\
\hline & $1 \mathrm{~m} \times 0.75 \mathrm{~m}$ & 105.3 & 6.0 & 43.7 & 29.0 & 5.3 & 79.5 \\
\hline & $1 \mathrm{~m} \times 0.5 \mathrm{~m}$ & 109.5 & 5.7 & 43.3 & 29.3 & 4.9 & 83.5 \\
\hline & CV (\%) & 15.8 & 12.0 & 9.4 & 11.9 & 25.9 & 19.2 \\
\hline & $P$ value & 0.32 & 0.61 & 0.78 & 0.68 & 0.75 & 0.33 \\
\hline & LSD (0.05) & 10.9 & 0.5 & 4.2 & 2.9 & 1.1 & 10.4 \\
\hline \multirow{6}{*}{20} & $1 \mathrm{~m} \times 1 \mathrm{~m}$ & 101.3 & 6.4 & 40.4 & 25.8 & 6.9 & 76.2 \\
\hline & $1 \mathrm{~m} \times 0.75 \mathrm{~m}$ & 106.9 & 6.1 & 41.4 & 26.4 & 7.1 & 81.2 \\
\hline & $1 \mathrm{~m} \times 0.5 \mathrm{~m}$ & 104.6 & 5.9 & 39.5 & 25.4 & 6.9 & 80.3 \\
\hline & CV (\%) & 9.8 & 10.9 & 9.8 & 9.0 & 25.4 & 12.6 \\
\hline & $P$ value & 0.48 & 0.05 & 0.39 & 0.66 & 0.96 & 0.43 \\
\hline & LSD (0.05) & 10.1 & 0.5 & 3.1 & 2.4 & 1.9 & 8.3 \\
\hline \multirow{6}{*}{24} & $1 \mathrm{~m} \times 1 \mathrm{~m}$ & 103.3 & 4.7 & 39.7 & 25.2 & 8.1 & 79.9 \\
\hline & $1 \mathrm{~m} \times 0.75 \mathrm{~m}$ & 108.8 & 4.7 & 40.3 & 25.8 & 7.9 & 83.6 \\
\hline & $1 \mathrm{~m} \times 0.5 \mathrm{~m}$ & 103.9 & 4.2 & 38.1 & 23.9 & 7.6 & 80.7 \\
\hline & CV (\%) & 9.4 & 20.4 & 10.6 & 9.2 & 26.2 & 10.7 \\
\hline & $P$ value & 0.49 & 0.16 & 0.44 & 0.26 & 0.78 & 0.64 \\
\hline & LSD (0.05) & 11.2 & 0.6 & 3.9 & 2.6 & 1.7 & 9.4 \\
\hline \multirow{6}{*}{28} & $1 \mathrm{~m} \times 1 \mathrm{~m}$ & 82.1 & 1.8 & 27.6 & 17.3 & 8.1 & 66.0 \\
\hline & $1 \mathrm{~m} \times 0.75 \mathrm{~m}$ & 88.9 & 1.9 & 29.9 & 18.7 & 8.1 & 71.4 \\
\hline & $1 \mathrm{~m} \times 0.5 \mathrm{~m}$ & 78.9 & 1.8 & 26.0 & 16.1 & 7.8 & 64.0 \\
\hline & CV (\%) & 17.5 & 24.3 & 22.7 & 19.9 & 24.8 & 17.1 \\
\hline & $P$ value & 0.53 & 0.82 & 0.42 & 0.43 & 0.87 & 0.58 \\
\hline & LSD (0.05) & 20.2 & 0.7 & 6.4 & 4.3 & 1.7 & 16.3 \\
\hline
\end{tabular}

$\mathrm{PH}=$ plant height, $\mathrm{NL} / \mathrm{P}=$ number of leaves, per plant, $\mathrm{LL}=$ leaf length, $\mathrm{LW}=$ leaf width, $\mathrm{PL}=$ petiole length, and $\mathrm{NS} / \mathrm{P}=$ number of suckers per plant. Significant $(P<0.05)$ factors are marked in bold.

higher number of leaves for accession BL/MS/16 was seen at 20 WAP in plants spaced at $1 \mathrm{~m} \times 1 \mathrm{~m}$. The number of suckers continued to increase until harvest with plants spaced at $1 \mathrm{~m} \times 1 \mathrm{~m}$ recording the highest for accession BL/MS/16 and $1 \mathrm{~m} \times 0.75 \mathrm{~m}$ for accession BL/MS/80.

3.2. Yield and Yield Components. The results of the effect of planting distance on the yield of taro are shown in Tables 5-7. Although both corm length and corm diameter were not significantly $(P \geq 0.05)$ influenced by planting distance, corm length and corm diameter were lower and larger, respectively, in closely spaced plants $(1 \mathrm{~m} \times 0.5 \mathrm{~m})$
(Table 5). Corm yield per plant was higher in plants spaced at $1 \mathrm{~m} \times 0.75 \mathrm{~m}$ although it did not show any significance. On the contrary corm yield ha ${ }^{-1}$ increased with the decrease in planting distance, hence planting at $1 \mathrm{~m} \times 0.5 \mathrm{~m}$ planting space produced the highest corm yield $\mathrm{ha}^{-1}$ and was significantly $(P \leq 0.001)$ higher than values obtained at $1 \mathrm{~m} \times 0.75 \mathrm{~m}$ and $1 \mathrm{~m} \times 1 \mathrm{~m}$ planting distances (Table 5). Planting distances had no effect on the harvest indices of the plants, but this was high in plants spaced at $1 \mathrm{~m} \times 0.5 \mathrm{~m}$.

Significant $(P \leq 0.05)$ accessions difference was identified among the accessions for corm length (Table 6). On the other hand, no significant $(P \geq 0.05)$ difference was observed for corm diameter, corm yield per plant, and yield ha ${ }^{-1}$. Overall, 
TABLE 2: Effect of different accessions of taro on growth parameters.

\begin{tabular}{|c|c|c|c|c|c|c|c|}
\hline Week & Accession & $\begin{array}{l}\mathrm{PH} \\
(\mathrm{cm})\end{array}$ & $\begin{array}{c}\mathrm{NL} / \\
\mathrm{P}\end{array}$ & $\begin{array}{l}\mathrm{LL} \\
(\mathrm{cm})\end{array}$ & $\begin{array}{l}\mathrm{LW} \\
(\mathrm{cm})\end{array}$ & $\begin{array}{l}\text { NS/ } \\
\mathrm{P}\end{array}$ & $\begin{array}{l}\mathrm{PL} \\
(\mathrm{cm})\end{array}$ \\
\hline \multirow{5}{*}{4} & $\mathrm{BL} / \mathrm{SM} / 80$ & 64.1 & 4.5 & 30.9 & 20.6 & 1.0 & 45.8 \\
\hline & $\mathrm{BL} / \mathrm{SM} / 16$ & 53.9 & 4.4 & 28.8 & 17.9 & 0.1 & 36.2 \\
\hline & CV (\%) & 4.6 & 11.5 & 6.0 & 6.1 & 21.5 & 3.4 \\
\hline & $P$ value & 0.04 & 0.78 & 0.27 & 0.11 & 0.04 & 0.02 \\
\hline & $\begin{array}{l}\text { LSD } \\
(0.05)\end{array}$ & 9.5 & 1.8 & 6.3 & 4.1 & 0.8 & 5.1 \\
\hline \multirow{5}{*}{8} & $\mathrm{BL} / \mathrm{SM} / 80$ & 98.1 & 5.3 & 41.2 & 29.0 & 2.9 & 73.2 \\
\hline & $\mathrm{BL} / \mathrm{SM} / 16$ & 83.6 & 5.7 & 41.1 & 26.9 & 2.6 & 57.6 \\
\hline & CV (\%) & 8.4 & 8.4 & 9.2 & 8.6 & 24.2 & 5.9 \\
\hline & $P$ value & 0.15 & 0.38 & 0.98 & 0.39 & 0.64 & 0.03 \\
\hline & $\begin{array}{c}\text { LSD } \\
(0.05)\end{array}$ & 26.9 & 1.6 & 13.3 & 8.5 & 2.8 & 13.6 \\
\hline \multirow{5}{*}{12} & $\mathrm{BL} / \mathrm{SM} / 80$ & 113.4 & 5.0 & 46.3 & 32.7 & 3.4 & 89.2 \\
\hline & $\mathrm{BL} / \mathrm{SM} / 16$ & 91.8 & 5.3 & 42.9 & 27.9 & 4.3 & 68.2 \\
\hline & CV (\%) & 12.2 & 1.9 & 9.4 & 9.4 & 21.5 & 8.8 \\
\hline & $P$ value & 0.17 & 0.06 & 0.42 & 0.18 & 0.30 & 0.07 \\
\hline & $\begin{array}{c}\text { LSD } \\
(0.05)\end{array}$ & 44.1 & 0.3 & 14.6 & 10.1 & 2.9 & 24.2 \\
\hline \multirow{5}{*}{16} & $\mathrm{BL} / \mathrm{SM} / 80$ & 116.9 & 5.4 & 44.4 & 30.6 & 3.9 & 90.6 \\
\hline & $\mathrm{BL} / \mathrm{SM} / 16$ & 94.2 & 6.4 & 41.9 & 27.0 & 6.3 & 68.8 \\
\hline & CV (\%) & 10.6 & 1.2 & 8.3 & 11.1 & 25.9 & 11.7 \\
\hline & $P$ value & 0.13 & 0.003 & 0.48 & 0.30 & 0.16 & 0.10 \\
\hline & $\begin{array}{l}\text { LSD } \\
(0.05)\end{array}$ & 39.5 & 0.3 & 12.6 & 11.2 & 4.7 & 32.8 \\
\hline \multirow{5}{*}{20} & $\mathrm{BL} / \mathrm{SM} / 80$ & 107.9 & 5.7 & 38.2 & 25.0 & 5.2 & 84.7 \\
\hline & $\mathrm{BL} / \mathrm{SM} / 16$ & 100.6 & 6.6 & 42.8 & 26.7 & 8.8 & 73.7 \\
\hline & CV (\%) & 9.0 & 5.2 & 8.0 & 8.5 & 18.1 & 9.7 \\
\hline & $P$ value & 0.44 & 0.07 & 0.23 & 0.46 & 0.07 & 0.22 \\
\hline & $\begin{array}{l}\text { LSD } \\
(0.05)\end{array}$ & 33.1 & 1.1 & 11.4 & 7.1 & 4.4 & 27.1 \\
\hline \multirow{5}{*}{24} & $\mathrm{BL} / \mathrm{SM} / 80$ & 105.6 & 3.9 & 36.8 & 23.9 & 5.6 & 83.3 \\
\hline & $\mathrm{BL} / \mathrm{SM} / 16$ & 105.1 & 5.2 & 41.9 & 25.2 & 10.1 & 79.5 \\
\hline & CV (\%) & 8.0 & 12.4 & 7.0 & 6.9 & 13.7 & 8.8 \\
\hline & $P$ value & 0.95 & 0.09 & 0.15 & 0.30 & 0.04 & 0.59 \\
\hline & $\begin{array}{l}\text { LSD } \\
(0.05)\end{array}$ & 29.5 & 1.9 & 9.7 & 6.0 & 3.8 & 25.3 \\
\hline \multirow{5}{*}{28} & $\mathrm{BL} / \mathrm{SM} / 80$ & 78.4 & 1.6 & 23.4 & 15.6 & 5.8 & 65.1 \\
\hline & $\mathrm{BL} / \mathrm{SM} / 16$ & 88.3 & 2.0 & 32.2 & 19.2 & 10.1 & 69.2 \\
\hline & CV (\%) & 11.9 & 14.4 & 9.6 & 11.0 & 6.6 & 13.0 \\
\hline & $P$ value & 0.35 & 0.22 & 0.05 & 0.15 & 0.01 & 0.62 \\
\hline & $\begin{array}{l}\text { LSD } \\
(0.05)\end{array}$ & 34.9 & 0.9 & 9.4 & 6.7 & 1.9 & 30.6 \\
\hline
\end{tabular}

$\mathrm{PH}=$ plant height, $\mathrm{NL} / \mathrm{P}=$ number of leaves, per plant, $\mathrm{LL}=$ leaf length, $\mathrm{LW}=$ leaf width, $\mathrm{PL}=$ petiole length, and $\mathrm{NS} / \mathrm{P}=$ number of suckers per plant. Significant $(P<0.05)$ factors are marked in bold.

accession $\mathrm{BL} / \mathrm{SM} / 80$ produced higher corm attributes and yield than accession BL/MS/16. Harvest index for the accessions was significant $(P \leq 0.05)$ with accession $\mathrm{BL} / \mathrm{MS} / 80$ again showing a higher value (Table 6).

The accession by planting distance interaction was significant $(P \leq 0.05$ and $P \leq 0.001)$ for some yield components (Table 7$)$. Significantly $(P \leq 0.05)$ higher corm length has shown accession $\mathrm{BL} / \mathrm{SM} / 80$ planted at $1 \mathrm{~m} \times 0.75 \mathrm{~m}$. Again, accession $\mathrm{BL} / \mathrm{SM} / 80$ planted at all the planting distance was seen to have higher corm length than accession BL/SM/16 planted at the same distance.
A significant $(P \leq 0.001)$ difference was observed for yield ha ${ }^{-1}$ (Table 7). There was a trend of increase in yield $\mathrm{ha}^{-1}$ as planting distance was decreased in all accessions. Accession $\mathrm{BL} / \mathrm{SM} / 80$ planted at $1 \mathrm{~m} \times 0.5 \mathrm{~m}$ produced the highest yield ha ${ }^{-1}$ among the accessions by planting distance combinations.

Accession $\mathrm{BL} / \mathrm{SM} / 80$ planted at $1 \mathrm{~m} \times 0.75 \mathrm{~m}$ produced the highest corm diameter and yield per plant although these were not significant $(P \geq 0.05)$ (Table 7$)$. Table 7 also shows the effect of the accession by planting distance combinations on harvest index. Significant $(P \leq 0.05)$ difference was observed with accession $\mathrm{BL} / \mathrm{SM} / 80$ recording the highest harvest index. Overall, the yield attributes of accession BL/ $\mathrm{SM} / 80$ planted at all the planting distances were better than those of accession $\mathrm{BL} / \mathrm{SM} / 16$ planted at the same planting distance.

The correlation of growth parameters, yield, and yield components is presented in Table 8 . The results indicate that growth parameters correlated significantly and positively with yield. Plant height correlated with corm diameter $\left(r=0.86^{* * *}\right)$, corm length $\left(r=0.70^{* * *}\right)$, yield/plant $\left(r=0.72^{* * *}\right)$, and yield/ha $\left(r=0.83^{* * *}\right)$. Leaf length also correlated with corm diameter $\left(r=0.71^{* * *}\right)$, corm length $\left(r=0.58^{* *}\right)$, yield/plant $\left(r=0.58^{* *}\right)$, and total yield/ha $\left(r=0.73^{* * *}\right)$. There was a significant and positive correlation between the number of leaves/plant and corm diameter $\left(r=0.51^{*}\right)$, yield/plant $\left(r=0.51^{*}\right)$, and total yield/ha $\left(r=0.53^{*}\right)$

\section{Discussion}

4.1. Growth. The growth of taro was not greatly affected by plant spacing as there were no significant differences $(P \leq 0.05)$ among the parameters except for 4 WAP where there existed a significant difference $(P \leq 0.05)$ in the plant spacing for plant height and petiole length, number of leaves, leaf length, and leaf width. Although there was no significance among the spacing, plants planted at lower spacing $(1 \mathrm{~m} \times 0.5 \mathrm{~m}$ and $1 \mathrm{~m} \times 0.75 \mathrm{~m})$ attained high growth (plant height, number of leaves/plant, and petiole length) than plants spaced at $1 \mathrm{~m} \times 1 \mathrm{~m}$ at the peak of vegetative growth (20 WAP). The results showed that growth (plant height, number of leaves, petiole length) increases with a decrease in plant spacing. Greater plant height at closer spacing may be due to more linear growth of plants as a result of higher plant density per unit area. Plant spacing had little or no effect on the growth of taro, and this may be because taro grows laterally instead of vertically. The nonsignificant effect of plant spacing on the growth of taro in this experiment agrees with the reports of $[18,19]$ who found nonsignificant effect of plant spacing (population density) on the growth of taro. The findings of the study differ from the reports of [20] and [21] who found that plant spacing (density) affected taro growth. In this study, a higher number of suckers were produced at 28 WAP ( 7 months after planting) and recorded by widely spaced plants $(1 \mathrm{~m} \times 1 \mathrm{~m})$. The number of suckers/ plant increased as plant spacing increases. The studies $[19,22,23]$ also collaborate the results of this study as they found that the number of suckers per plant decreased with 
TABLE 3: Effect of planting distance by accession interaction at 4, 8, 12, and 16 weeks after planting (WAP).

\begin{tabular}{|c|c|c|c|c|c|c|c|}
\hline Accession & Treatment & $\mathrm{PH}(\mathrm{cm})$ & $\mathrm{NL} / \mathrm{P}$ & $\mathrm{LL}(\mathrm{cm})$ & $\mathrm{LW}(\mathrm{cm})$ & $\mathrm{NS} / \mathrm{P}$ & $\mathrm{PL}(\mathrm{cm})$ \\
\hline & & & 4 WAP & & & & \\
\hline $\mathrm{BL} / \mathrm{SM} / 80$ & $1 \mathrm{~m} \times 1 \mathrm{~m}$ & 59.1 & 4.5 & 28.3 & 18.9 & 0.5 & 42.5 \\
\hline $\mathrm{BL} / \mathrm{SM} / 80$ & $1 \mathrm{~m} \times 0.75 \mathrm{~m}$ & 64.6 & 4.5 & 32.1 & 21.3 & 1.1 & 46.3 \\
\hline $\mathrm{BL} / \mathrm{SM} / 80$ & $1 \mathrm{~m} \times 0.5 \mathrm{~m}$ & 68.6 & 4.6 & 32.5 & 21.7 & 1.4 & 48.5 \\
\hline $\mathrm{BL} / \mathrm{SM} / 16$ & $1 \mathrm{~m} \times 1 \mathrm{~m}$ & 49.4 & 4.4 & 26.9 & 16.6 & 0 & 32.9 \\
\hline $\mathrm{BL} / \mathrm{SM} / 16$ & $1 \mathrm{~m} \times 0.75 \mathrm{~m}$ & 54.3 & 4.0 & 28.6 & 17.8 & 0.3 & 36.3 \\
\hline $\mathrm{BL} / \mathrm{SM} / 16$ & $1 \mathrm{~m} \times 0.5 \mathrm{~m}$ & 58.3 & 4.7 & 30.8 & 19.3 & 0.1 & 39.5 \\
\hline $\mathrm{CV}(\%)$ & & 8.8 & 6.4 & 9.0 & 9.3 & 23.4 & 8.5 \\
\hline$P$ value & & 0.99 & 0.24 & 0.79 & 0.82 & 0.59 & 0.97 \\
\hline \multirow[t]{2}{*}{ LSD (0.05) } & & 11.8 & 1.8 & 7.2 & 4.7 & 1.2 & 7.1 \\
\hline & & & $8 \mathrm{WAP}$ & & & & \\
\hline $\mathrm{BL} / \mathrm{SM} / 80$ & $1 \mathrm{~m} \times 1 \mathrm{~m}$ & 92.1 & 4.9 & 38.7 & 26.8 & 2.3 & 70.0 \\
\hline $\mathrm{BL} / \mathrm{SM} / 80$ & $1 \mathrm{~m} \times 0.75 \mathrm{~m}$ & 100.3 & 5.6 & 41.8 & 29.7 & 3.5 & 71.5 \\
\hline $\mathrm{BL} / \mathrm{SM} / 80$ & $1 \mathrm{~m} \times 0.5 \mathrm{~m}$ & 102.0 & 5.3 & 43.1 & 30.5 & 3.1 & 78.0 \\
\hline $\mathrm{BL} / \mathrm{SM} / 16$ & $1 \mathrm{~m} \times 1 \mathrm{~m}$ & 78.9 & 5.6 & 39.6 & 26.2 & 2.4 & 52.4 \\
\hline $\mathrm{BL} / \mathrm{SM} / 16$ & $1 \mathrm{~m} \times 0.75 \mathrm{~m}$ & 82.3 & 5.5 & 40.4 & 26.2 & 2.7 & 57.8 \\
\hline $\mathrm{BL} / \mathrm{SM} / 16$ & $1 \mathrm{~m} \times 0.5 \mathrm{~m}$ & 89.6 & 6.0 & 43.3 & 28.2 & 2.8 & 62.5 \\
\hline $\mathrm{CV}(\%)$ & & 9.9 & 10.0 & 9.6 & 10.8 & 24.5 & 9.4 \\
\hline$P$ value & & 0.85 & 0.45 & 0.88 & 0.70 & 0.79 & 0.86 \\
\hline \multirow[t]{2}{*}{ LSD (0.05) } & & 29.0 & 1.7 & 14.1 & 9.2 & 3.1 & 15.8 \\
\hline & & & $12 \mathrm{WAP}$ & & & & \\
\hline $\mathrm{BL} / \mathrm{SM} / 80$ & $1 \mathrm{~m} \times 1 \mathrm{~m}$ & 112.6 & 5.1 & 45.1 & 32.5 & 2.9 & 86 \\
\hline $\mathrm{BL} / \mathrm{SM} / 80$ & $1 \mathrm{~m} \times 0.75 \mathrm{~m}$ & 115.9 & 5.1 & 46.7 & 32.9 & 3.8 & 89.6 \\
\hline $\mathrm{BL} / \mathrm{SM} / 80$ & $1 \mathrm{~m} \times 0.5 \mathrm{~m}$ & 111.8 & 4.8 & 47 & 32.6 & 3.7 & 92.1 \\
\hline $\mathrm{BL} / \mathrm{SM} / 16$ & $1 \mathrm{~m} \times 1 \mathrm{~m}$ & 87.7 & 5.3 & 40.9 & 26.8 & 3.8 & 63.0 \\
\hline $\mathrm{BL} / \mathrm{SM} / 16$ & $1 \mathrm{~m} \times 0.75 \mathrm{~m}$ & 87.2 & 5.5 & 42 & 27.6 & 4.5 & 67.5 \\
\hline $\mathrm{BL} / \mathrm{SM} / 16$ & $1 \mathrm{~m} \times 0.5 \mathrm{~m}$ & 100.6 & 5.1 & 45.7 & 29.6 & 4.5 & 74.0 \\
\hline $\mathrm{CV}(\%)$ & & 11.7 & 8.0 & 9.0 & 9.9 & 28.7 & 10.3 \\
\hline$P$ value & & 0.45 & 0.91 & 0.73 & 0.72 & 0.99 & 0.9 \\
\hline \multirow[t]{2}{*}{ LSD $(0.05)$} & & 46.1 & 0.7 & 15.3 & 10.6 & 3.2 & 26.1 \\
\hline & & & $16 \mathrm{WAP}$ & & & & \\
\hline $\mathrm{BL} / \mathrm{SM} / 80$ & $1 \mathrm{~m} \times 1 \mathrm{~m}$ & 113.4 & 5.3 & 44.3 & 30.3 & 3.5 & 87.1 \\
\hline $\mathrm{BL} / \mathrm{SM} / 80$ & $1 \mathrm{~m} \times 0.75 \mathrm{~m}$ & 117.9 & 5.7 & 45.5 & 31.6 & 4.6 & 91.4 \\
\hline $\mathrm{BL} / \mathrm{SM} / 80$ & $1 \mathrm{~m} \times 0.5 \mathrm{~m}$ & 119.4 & 5.0 & 43.5 & 30.0 & 3.7 & 93.3 \\
\hline $\mathrm{BL} / \mathrm{SM} / 16$ & $1 \mathrm{~m} \times 1 \mathrm{~m}$ & 90.2 & 6.5 & 40.6 & 26.1 & 6.7 & 65.3 \\
\hline $\mathrm{BL} / \mathrm{SM} / 16$ & $1 \mathrm{~m} \times 0.75 \mathrm{~m}$ & 92.7 & 6.2 & 41.9 & 26.5 & 6.0 & 67.6 \\
\hline $\mathrm{BL} / \mathrm{SM} / 16$ & $1 \mathrm{~m} \times 0.5 \mathrm{~m}$ & 99.6 & 6.5 & 43.2 & 28.5 & 6.2 & 73.6 \\
\hline $\mathrm{CV}(\%)$ & & 7.8 & 6.9 & 7.3 & 7.6 & 16 & 9.8 \\
\hline$P$ value & & 0.85 & 0.16 & 0.59 & 0.37 & 0.22 & 0.90 \\
\hline LSD (0.05) & & 40.4 & 0.7 & 13.0 & 11.4 & 4.7 & 33.8 \\
\hline
\end{tabular}

$\mathrm{PH}=$ plant height, $\mathrm{NL} / \mathrm{P}=$ number of leaves, per plant, $\mathrm{LL}=$ leaf length, $\mathrm{LW}=$ leaf width, $\mathrm{PL}=$ petiole length, and $\mathrm{NS} / \mathrm{P}=$ number of suckers per plant.

decrease in plant spacing. The increase in sucker number with an increase in plant spacing may be due to greater number of available assimilates for below ground and the availability of more nutrients, moisture, and low competition for light at higher spaced plants.

4.2. Yield. Plants spacing had no effect on corm yield per plant although higher corm yield per plant was recorded by higher plant spacing $(1 \mathrm{~m} \times 1 \mathrm{~m}$ and $1 \mathrm{~m} \times 0.75 \mathrm{~m})$. The observed decrease in corm yield per stand at closer planting space is in agreement with the findings of [24, 25]. However, it has been reported that corm yield is a function of the number of corms and weight of corms per planting hill [26]. Calculated on a hectare basis, corm yield $\mathrm{ha}^{-1}$ was found to increase as planting distance decreases. In this study, the highest corm yield $\mathrm{ha}^{-1}$ was observed in the closely planting distance $(1 \mathrm{~m} \times 0.5 \mathrm{~m})$. It is noteworthy that the yields observed in closely spaced plants $(1 \mathrm{~m} \times 0.5 \mathrm{~m}$ and $1 \mathrm{~m} \times 0.75 \mathrm{~m}$ ) were equal or even greater than the African and world average of 5.9 and $6.6 \mathrm{t} \cdot \mathrm{ha}^{-1}$, respectively [26], and were obtained without additional production inputs (water, fertilizers, etc.). The results showed that the total yield in taro is a function of the number of corms produced per unit area rather than the size of the individual corm. This suggests that the increase in total corm yield was due to the higher number of plants per unit area, which intercepts solar radiation and thereby enhances photosynthesis on a unit area basis. It is also possible that, at lower plant spacing, there was an added advantage of full-ground cover which effectively suppressed weeds, hence, contributing to greater yield. The finding of the study is in agreement with previous reports by $[15,27-29]$. 
TABLE 4: Effect of planting distance by accession interaction at 20, 24, and 28 weeks after planting (WAP).

\begin{tabular}{|c|c|c|c|c|c|c|c|}
\hline Accession & Treatment & $\mathrm{PH}(\mathrm{cm})$ & $\mathrm{NL} / \mathrm{P}$ & $\mathrm{LL}(\mathrm{cm})$ & $\mathrm{LW}(\mathrm{cm})$ & $\mathrm{NS} / \mathrm{P}$ & $\mathrm{PL}(\mathrm{cm})$ \\
\hline & & & $20 \mathrm{WAP}$ & & & & \\
\hline $\mathrm{BL} / \mathrm{SM} / 80$ & $1 \mathrm{~m} \times 1 \mathrm{~m}$ & 104.6 & 6.0 & 37.8 & 24.8 & 5.3 & 81.5 \\
\hline $\mathrm{BL} / \mathrm{SM} / 80$ & $1 \mathrm{~m} \times 0.75 \mathrm{~m}$ & 112.0 & 5.8 & 40.1 & 26.2 & 5.1 & 87.7 \\
\hline $\mathrm{BL} / \mathrm{SM} / 80$ & $1 \mathrm{~m} \times 0.5 \mathrm{~m}$ & 107.3 & 5.3 & 36.6 & 24.2 & 5.1 & 85.0 \\
\hline $\mathrm{BL} / \mathrm{SM} / 16$ & $1 \mathrm{~m} \times 1 \mathrm{~m}$ & 98.1 & 6.8 & 43.0 & 26.9 & 8.6 & 70.9 \\
\hline $\mathrm{BL} / \mathrm{SM} / 16$ & $1 \mathrm{~m} \times 0.75 \mathrm{~m}$ & 101.8 & 6.5 & 42.7 & 26.6 & 9.1 & 74.7 \\
\hline $\mathrm{BL} / \mathrm{SM} / 16$ & $1 \mathrm{~m} \times 0.5 \mathrm{~m}$ & 101.9 & 6.7 & 42.5 & 26.6 & 8.7 & 75.5 \\
\hline $\mathrm{CV}(\%)$ & & 7.3 & 5.8 & 5.7 & 7.1 & 21.2 & 8.4 \\
\hline$P$ value & & 0.85 & 0.23 & 0.45 & 0.62 & 0.93 & 0.90 \\
\hline \multirow[t]{2}{*}{ LSD (0.05) } & & 34.0 & 1.2 & 11.7 & 8.0 & 4.8 & 28.0 \\
\hline & & & $24 \mathrm{WAP}$ & & & & \\
\hline $\mathrm{BL} / \mathrm{SM} / 80$ & $1 \mathrm{~m} \times 1 \mathrm{~m}$ & 103.1 & 3.9 & 36.9 & 23.9 & 5.6 & 80.9 \\
\hline $\mathrm{BL} / \mathrm{SM} / 80$ & $1 \mathrm{~m} \times 0.75 \mathrm{~m}$ & 110.7 & 4.3 & 39 & 25.5 & 6.1 & 86.7 \\
\hline $\mathrm{BL} / \mathrm{SM} / 80$ & $1 \mathrm{~m} \times 0.5 \mathrm{~m}$ & 102.9 & 3.5 & 34.6 & 22.6 & 5.3 & 82.2 \\
\hline $\mathrm{BL} / \mathrm{SM} / 16$ & $1 \mathrm{~m} \times 1 \mathrm{~m}$ & 103.5 & 5.5 & 42.5 & 26.5 & 10.6 & 78.8 \\
\hline $\mathrm{BL} / \mathrm{SM} / 16$ & $1 \mathrm{~m} \times 0.75 \mathrm{~m}$ & 106.9 & 5.2 & 41.6 & 26.1 & 9.7 & 80.6 \\
\hline $\mathrm{BL} / \mathrm{SM} / 16$ & $1 \mathrm{~m} \times 0.5 \mathrm{~m}$ & 104.9 & 5.0 & 41.5 & 25.1 & 9.9 & 79.2 \\
\hline CV (\%) & & 8.0 & 9.6 & 7.5 & 7.9 & 16.7 & 8.6 \\
\hline$P$ value & & 0.83 & 0.39 & 0.48 & 0.62 & 0.68 & 0.88 \\
\hline \multirow[t]{2}{*}{ LSD (0.05) } & & 30.9 & 2.0 & 10.3 & 6.5 & 4.1 & 26.5 \\
\hline & & & $28 \mathrm{WAP}$ & & & & \\
\hline $\mathrm{BL} / \mathrm{SM} / 80$ & $1 \mathrm{~m} \times 1 \mathrm{~m}$ & 76.9 & 1.5 & 22.9 & 15.1 & 5.7 & 64.0 \\
\hline $\mathrm{BL} / \mathrm{SM} / 80$ & $1 \mathrm{~m} \times 0.75 \mathrm{~m}$ & 86.0 & 1.8 & 26.2 & 17.7 & 6.1 & 70.5 \\
\hline $\mathrm{BL} / \mathrm{SM} / 80$ & $1 \mathrm{~m} \times 0.5 \mathrm{~m}$ & 72.4 & 1.6 & 21.2 & 14 & 5.6 & 60.8 \\
\hline $\mathrm{BL} / \mathrm{SM} / 16$ & $1 \mathrm{~m} \times 1 \mathrm{~m}$ & 87.3 & 2.1 & 32.3 & 19.5 & 10.5 & 68.0 \\
\hline $\mathrm{BL} / \mathrm{SM} / 16$ & $1 \mathrm{~m} \times 0.75 \mathrm{~m}$ & 92.0 & 2.1 & 33.6 & 19.8 & 10.1 & 72.2 \\
\hline $\mathrm{BL} / \mathrm{SM} / 16$ & $1 \mathrm{~m} \times 0.5 \mathrm{~m}$ & 85.6 & 1.9 & 30.8 & 18.3 & 9.9 & 67.3 \\
\hline CV (\%) & & 18.2 & 27.6 & 17.4 & 18.8 & 15.9 & 18.2 \\
\hline$P$ value & & 0.92 & 0.75 & 0.91 & 0.79 & 0.88 & 0.95 \\
\hline LSD (0.05) & & 40.0 & 1.2 & 11.5 & 8.0 & 2.6 & 34.2 \\
\hline
\end{tabular}

$\mathrm{PH}=$ plant height, $\mathrm{NL} / \mathrm{P}=$ number of leaves, per plant, $\mathrm{LL}=$ leaf length, $\mathrm{LW}=$ leaf width, $\mathrm{PL}=$ petiole length, and $\mathrm{NS} / \mathrm{P}=$ number of suckers per plant.

TABLE 5: Effect of different planting distances on yield attributes of taro.

\begin{tabular}{lccccc}
\hline Planting distance & Corm length $(\mathrm{cm})$ & Corm diameter $(\mathrm{cm})$ & Yield per plant $(\mathrm{kg})$ & Yield/ha $(\mathrm{t} / \mathrm{ha})$ & Harvest index $(\%)$ \\
\hline $1 \mathrm{~m} \times 1 \mathrm{~m}$ & 14.5 & 28.9 & 0.59 & 5.9 & 82.8 \\
$1 \mathrm{~m} \times 0.75 \mathrm{~m}$ & 15.4 & 30.2 & 0.63 & 0.4 & 71.6 \\
$1 \mathrm{~m} \times 0.5 \mathrm{~m}$ & 14.4 & 30.4 & 0.58 & 27.6 & 85.9 \\
$\mathrm{CV}(\%)$ & 15.2 & 11.7 & 0.63 & 0.001 & 13.6 \\
$P$ value & 0.23 & 0.41 & 0.12 & 1.7 & 0.07 \\
LSD $(\mathbf{0 . 0 5})$ & 1.3 & 2.7 & & & 12.8 \\
\hline
\end{tabular}

TABLE 6: Effect of different accession on yield attributes of taro.

\begin{tabular}{lccccc}
\hline Accession & Corm length $(\mathrm{cm})$ & Corm diameter $(\mathrm{cm})$ & Yield per plant $(\mathrm{kg})$ & Yield/ha $(\mathrm{t} / \mathrm{ha})$ & Harvest index $(\%)$ \\
\hline $\mathrm{BL} / \mathrm{SM} / 80$ & 16.5 & 32.2 & 0.72 & 10.2 & 86.5 \\
$\mathrm{BL} / \mathrm{SM} / 16$ & 12.9 & 27.4 & 0.48 & 17.7 & 73.7 \\
$\mathrm{CV}(\%)$ & 6.9 & 4.6 & 0.1 & 0.12 & 1.4 \\
$P$ value & 0.05 & 0.10 & 0.42 & 5.3 & 0.005 \\
LSD (0.05) & 3.6 & 7.1 & 4.0 & \\
\hline
\end{tabular}

Results of the correlation analysis revealed a strong positive and significant correlation between growth parameters, yield, and yield components. This indicates that increasing these growth parameters increases yield. The strong positive correlation of the traits shows a great agronomic potential of the two cultivars used in the study. The studies [30-32] reported a positive correlation for growth and yield parameters of taro. Other authors have also reported the interdependence between genetic variables and yields. 
TABLE 7: Effect of planting distance by accession interaction on yield attributes of taro.

\begin{tabular}{|c|c|c|c|c|c|c|}
\hline Accession & Planting distance & Corm length $(\mathrm{cm})$ & Corm diameter $(\mathrm{cm})$ & Yield per plant $(\mathrm{kg})$ & Yield/ha (t/ha) & Harvest index (\%) \\
\hline $\mathrm{BL} / \mathrm{SM} / 80$ & $1 \mathrm{~m} \times 1 \mathrm{~m}$ & 16.7 & 31.8 & 0.73 & 7.3 & 93.1 \\
\hline $\mathrm{BL} / \mathrm{SM} / 80$ & $1 \mathrm{~m} \times 0.75 \mathrm{~m}$ & 17.5 & 32.6 & 0.77 & 10.3 & 83.9 \\
\hline $\mathrm{BL} / \mathrm{SM} / 80$ & $1 \mathrm{~m} \times 0.5 \mathrm{~m}$ & 15.3 & 32.1 & 0.65 & 13.0 & 92.9 \\
\hline $\mathrm{BL} / \mathrm{SM} / 16$ & $1 \mathrm{~m} \times 1 \mathrm{~m}$ & 12.3 & 25.9 & 0.45 & 4.5 & 68.0 \\
\hline $\mathrm{BL} / \mathrm{SM} / 16$ & $1 \mathrm{~m} \times 0.75 \mathrm{~m}$ & 13.2 & 27.7 & 0.49 & 6.5 & 62.1 \\
\hline $\mathrm{BL} / \mathrm{SM} / 16$ & $1 \mathrm{~m} \times 0.5 \mathrm{~m}$ & 13.4 & 28.6 & 0.51 & 10.2 & 83.7 \\
\hline CV (\%) & & 6.7 & 6.7 & 14.3 & 14.6 & 12.0 \\
\hline$P$ value & & 0.05 & 0.60 & 0.31 & 0.001 & 0.05 \\
\hline LSD $(P \leq 0.05)$ & & 3.7 & 7.4 & 0.40 & 5.5 & 15.3 \\
\hline
\end{tabular}

TABle 8: Association of taro growth, yield, and yield components.

\begin{tabular}{|c|c|c|c|c|c|c|c|c|c|c|c|}
\hline Characters & $\mathrm{CD}$ & $\mathrm{CL}$ & $\mathrm{HI}$ & LL & LW & $\mathrm{NL}$ & NS & $\mathrm{PH}$ & $\mathrm{PL}$ & $\mathrm{Y} / \mathrm{P}$ & Y/ha \\
\hline $\mathrm{CD}$ & 1 & & & & & & & & & & \\
\hline $\mathrm{CL}$ & $0.849^{* * *}$ & 1 & & & & & & & & & \\
\hline HI & 0.346 & 0.405 & 1 & & & & & & & & \\
\hline LL & $0.705^{* * *}$ & $0.575^{* *}$ & 0.223 & 1 & & & & & & & \\
\hline LW & $0.831^{* * *}$ & $0.694^{* * *}$ & 0.313 & $0.968^{* * *}$ & 1 & & & & & & \\
\hline NL & $0.508^{*}$ & 0.335 & 0.399 & $0.602^{* *}$ & $0.592^{* *}$ & 1 & & & & & \\
\hline NS & $0.752^{* * *}$ & $0.671^{* *}$ & 0.214 & $0.697^{* * *}$ & $0.792^{* * *}$ & 0.430 & 1 & & & & \\
\hline $\mathrm{PH}$ & $0.861^{* * *}$ & $0.702^{* * *}$ & 0.354 & $0.893^{* * *}$ & $0.968^{* * *}$ & $0.508^{*}$ & $0.844^{* * *}$ & 1 & & & \\
\hline PL & $0.906^{* * *}$ & $0.779^{* * *}$ & 0.419 & $0.793^{* * *}$ & $0.914^{* * *}$ & $0.466^{*}$ & $0.856^{* * *}$ & $0.975^{* * *}$ & 1 & & \\
\hline $\mathrm{Y} / \mathrm{P}$ & $0.953^{* * *}$ & $0.879^{* * *}$ & 0.296 & $0.575^{* *}$ & $0.709^{* * *}$ & $0.505^{*}$ & $0.681^{* * *}$ & $0.720^{* * *}$ & $0.795^{* * *}$ & 1 & \\
\hline Y/ha & $0.766^{* * *}$ & $0.523^{*}$ & 0.405 & $0.734^{* * *}$ & $0.796^{* * *}$ & $0.529^{*}$ & $0.677^{* *}$ & $0.832^{* * *}$ & $0.841^{* * *}$ & $0.627^{* *}$ & 1 \\
\hline
\end{tabular}

$\mathrm{CD}=$ corm diameter, $\mathrm{CL}=$ corm length, $\mathrm{HI}=$ harvest index, $\mathrm{LL}=$ leaf length, $\mathrm{LW}=$ leaf width, $\mathrm{NL}=$ number of leaves, $\mathrm{NS}=$ number of suckers, $\mathrm{PH}=$ plant height, $\mathrm{PL}=$ petiole length, $\mathrm{Y} / \mathrm{P}=$ yield per plant, and $\mathrm{Y} / \mathrm{ha}=$ yield per hectare. ${ }^{*} P \leq 0.05,{ }^{* *} P \leq 0.01$, and ${ }^{* * *} P \leq 0.001$.

\section{Conclusion}

In this study, spacing played a significant role in determining the overall performance of taro. Wider spacing increased the corm yield of individual taro plants whereas narrow spacing increased the total corm yield of taro per unit area. Based on the results from this study, planting at the closer spacing $1 \mathrm{~m} \times 0.5 \mathrm{~m}$ which produced the highest corm yield $\mathrm{ha}^{-1}$ in all accessions is recommended to farmers. Accession $\mathrm{BL} / \mathrm{SM} / 80$ which also produced high corm yield ha ${ }^{-1}$ is also recommended to farmers in the area.

\section{Data Availability}

The data used to support the findings of this study are available from the corresponding author upon request.

\section{Conflicts of Interest}

The authors declare that they have no conflicts of interest regarding the publication of this study.

\section{Acknowledgments}

The authors hereby acknowledge the support of the International Network for Edible Aroids (INEA) for introducing the accessions in Ghana.

\section{References}

[1] R. J. Henry, Plant Genotyping: The DNA Fingerprinting of Plants, CAB Publishing, Southern Cross University, Australia, 2001.

[2] V. Lebot, Tropical Root and Tuber Crops: Cassava, Sweet Potato, Yams and Aroids, pp. 279-349, CABI, Cambridge, UK, 2009.

[3] TaroGen, "AusAID/SPC taro genetic Resources: conservation and utilization project annual report 1999/2000," Secretariat of the Pacific Community, Noumea, New Caledonia, 2000.

[4] B. E. V. Wyk, Food Plants of the World: Identification, Culinary Uses and Nutritional Value, p. 275, Times Editions-Marshall Cavendish, Singapore, 2005.

[5] G. Vinning, Select Markets for Taro, Sweet Potato and Yam, RIRDC, Australia, Project No. UCQ-13A, 2003.

[6] P. A. Revill, G. V. H. Jackson, G. J. Hafner et al., "Incidence and distribution of viruses of taro (Colocasia esculenta) in Pacific island countries," Australasian Plant Pathology, vol. 34, no. 3, pp. 327-331, 2005.

[7] R. S. Misra, S. Sriram, J. N. Govil, J. Pandey, B. G. Shivakumar, and V. K. Singh, "Medicinal value and export potential of tropical tuber crop," Crop Improvement Production Technology Trade and Commerce, vol. 5, pp. 376-386, 2002.

[8] W. Nip, J. Muchille, T. Cai, and J. H. Moy, "Nutritive and non-nutritive constituents in taro (Colocasia esculenta (L.) Schott) from American Samoa," Journal of Hawaiian and Pacific Agriculture, vol. 2, pp. 1-5, 1989.

[9] K. Ofori, "Comparison of taro production and constraints between west Africa and the Pacific," in Proceedings of the Third Taro Symposium, pp. 21-23, Nadi, Fiji, May 2003. 
[10] FAO, "FAO bulletin of statistics," 2018, http://faostat.fao.org.

[11] P. E. Ogbonna, K. O. Orji, N. J. Nweze, and P. Opata, "Effect of planting space on plant population at harvest and tuber yield in taro (Colocasia esculenta L)," African Journal of Agricultural Research, vol. 10, no. 5, pp. 308-316, 2015.

[12] G. R. Squire, The Physiology of Tropical Crop Production, p. 229, CAB International, Wallingford, UK, 1990.

[13] J. A. Barden, R. G. Halfacre, and D. J. Parish, Plant Science, p. 551, McGraw-Hill, New York, NY, USA, 1989.

[14] P. E. Ogbonna and I. U. Obi, "The influence of poultry manure application and plant density on the growth and yield of egusi melon (Colocynthis citrullus) on the Nsukka plains of south eastern Nigeria," Agro-Science, vol. 1, no. 1, pp. 122-129, 2000.

[15] B. Osundare, "Effects of fertilizer types and varying population on the performance of cocoyam," Moor Journal of Agricultural Research, vol. 5, no. 1, pp. 7-12, 2004.

[16] Meteorological Service Department, Annual Report, East Akim Municipal Assembly, East Akim, Ghana, 2014.

[17] Analytical Software, Statistix 9 User Manual, Analytical Software, Tallahassee, FL, USA, 2008.

[18] K. Kamal, S. Deo, A. K. Thakur, R. R. Kanwar, J. Singh, and R. R. Saxena, "Effects of planting techniques and spacing on corm and cormel yield of bunda (Colocasia esculenta Var. esculenta) under Bastan Plateau of Chhattisgarh, India," Plant Achives, vol. 16, no. 2, pp. 659-666, 2016, http://www. plantarchives.org/PDF\%2016.

[19] T. Mulugeta, T. Bizuayehu, and G. Yasin, "Effects of type of planting material and population density on corm yield and yield components of taro (Colocasia esculenta L.)," Journal of Biology, Agriculture and Healthcare, vol. 4, no. 17, 2014.

[20] S. S. Gill, B. S. Randhawa, and S. S. Saini, "Optimizing the agronomic requirements of taro (Colocosia esculenta.esc) for Punjab," Indian Journal of Agronomy, vol. 50, no. 2, pp. 170-172, 2005.

[21] A. A. Abd-Ellatif, A. El-Ezz, E. Shehata, and S. M. Yousseff, "Effect of planting dates and intra-row spacing on growth, yield, and quality of taro," Research Journal of Agricultureand Biological Science, vol. 6, no. 6, pp. 806-814, 2010.

[22] P. A. Gendua, J. B. Risimeri, and J. B. Maima, "The effect of planting density on the marketable corm yield of taro (Colocasia esculenta (L.) Schott)," pp. 764-767, Bubia Resarch Station, National Agricultural Research Institute (NARI) PNG, Morobe, Papua New Guinea, 2000.

[23] C. R. Gregory, "Dasheen (Colocasia esculenta (L) Schott var. esculenta): research in Dominica to address constraints to production," A Compilation of CARDI Research Papers, CARDI Review, pp. 2-9, Caribbean Agricultural Research and Development Institute, St. Augustin, Trinidad and Tobago, 2004, http://www.cardi.org/images/public/re.

[24] J. J. C. Scheffer, J. A. Douglas, and C. M. Triggs, "Factors affecting the production and quality of Japanese taro cormels," Acta Horticulturae, vol. 670, pp. 167-172, 2005.

[25] R. Tumuhimbise, H. Talwana, D. Osiru et al., "Growth and development of wetland-grown taro under different plant populations and seedbed types in Uganda," African Crop Science Journal, vol. 17, no. 1, pp. 49-60, 2009.

[26] A. A. Kader and R. S. Rolle, The Role of Post-harvest Management in Assuring the Quality and Safety of Horticultural Produce, Food \& Agriculture Organization, Rome, Italy, 2004.

[27] M. D. Atiquzzaman, Effect of Spacing on the Growth and Yield of Mukhi Kachu (Colocasia esculenta) cv. Pancho Mukhi, Department of Crop Botany, Bangladesh, 2007.

[28] H. L. Talwana, R. Tumuhimbise, and D. S. O. Osiru, "Comparative performance of wetland taro grown in upland production systems as influenced by different plant densities and seedbed preparation in Uganda," Journal of Root Crops, vol. 36, no. 1, pp. 65-71, 2010.

[29] N. A. H. Zárate, M. d. C. Vieira, E. J. Rosa Júnior, J. C. Alves, and J. C. Alves, "Populações de plantas e doses de nitrogênio na produção de rizomas de taro "Macaquinho"," Ciência $e$ Agrotecnologia, vol. 28, no. 5, p. 1190, 2004.

[30] S. Fantaw, A. Nebiyu, and T. Mulualem, "Estimates of genetic components for yield and yield related traits of Tannia (Xanthosoma sagitifolium (L.) Scott) genotypesat Jimma Southwest Ethiopia," African Journal of Agricultural Research, vol. 10, pp. 23-30, 2014.

[31] P. E. Norman, A. A. Beah, J. F. Bebeley, and E. F. Sellu, "Assessment of agro morphological diversity and affinities in yautia species from Sierra Leone," International Journal of Biodiversity Conservation, vol. 7, no. 10, pp. 408-419, 2015.

[32] K. Paul and M. Bari, "Estimates of genetic components for yield and related traits in cocoyam," The Agriculturists, vol. 10, no. 2, pp. 127-132, 2012. 